INPLASY

PROTOCOL

To cite: Cheng et al. Highdensity lipoprotein Cholesterol Efflux Capacity and the Risk of Cardiovascular Diseases: A Systematic Review and Metaanalysis. Inplasy protocol 202170006. doi: 10.37766/inplasy2021.7.0006

Received: 02 July 2021

Published: 02 July 2021

Corresponding author:

Petra Buettner

Petra.Buettner@medizin.uni-leipzig.de

Author Affiliation:

Leipzig Heart Center.

Support: None.

Review Stage at time of this submission: Piloting of the study selection process.

Conflicts of interest:

None declared.

\section{High-density lipoprotein Cholesterol Efflux Capacity and the Risk of Cardiovascular Diseases: A Systematic Review and Meta-analysis}

Cheng, W1; Boettner, J2; Fischer-Schaepman, T3; Werner, S4; Kricke, $\mathrm{A}^{5}$; Thiele, $\mathrm{H}^{6}$; Buettner, $\mathrm{P}^{7}$.

Review question / Objective: Review question: many studies have indicated that a negative association between HDL cholesterol efflux capacity (CEC) and cardiovascular diseases. However, cardiovascular disease as a general term for a group of cardiovascular-related diseases, whether there are differences between diseases. Besides, it remains unknown whether HDL-CEC is dose-responsive to cardiovascular disease risk. Objective: This paper is aimed to systematically investigate the association between HDL-CEC and cardiovascular diseases.

Condition being studied: Risk of Cardiovascular Diseases.

INPLASY registration number: This protocol was registered with the International Platform of Registered Systematic Review and Meta-Analysis Protocols (INPLASY) on 02 July 2021 and was last updated on 02 July 2021 (registration number INPLASY202170006).

\section{INTRODUCTION}

Review question / Objective: Review question: many studies have indicated that a negative association between HDL cholesterol efflux capacity (CEC) and cardiovascular diseases. However, cardiovascular disease as a general term for a group of cardiovascular-related diseases, whether there are differences between diseases. Besides, it remains unknown whether HDL-CEC is doseresponsive to cardiovascular disease risk. Objective: This paper is aimed to systematically investigate the association 
between HDL-CEC and cardiovascular diseases.

Condition being studied: Risk of Cardiovascular Diseases.

\section{METHODS}

Participant or population: Cardiovascular disease or General population.

Intervention: HDL-CEC level.

Comparator: healthy population or different caro-diseases; control.

Study designs to be included: cohort studies/ case-control; prospective cohort studies.

Eligibility criteria: 1 . Study population aged $>18$ years with a history of cardiovascular disease. 2. The exposure of study was HDL-CEC. 3. The intervention of study was different levels of HDL-CEC. 4. The control group was patients with cardiovascular disease or healthy population. 5 . The study outcomes were the risk of cardiovascularrelated disease.

Information sources: Web of science, pubmed, and Embase were searched from 2nd, July, 2021. In addition, previous reviews and meta-analysis were included for make full use.

Main outcome(s): The study outcomes were the risk of cardiovascular-related disease.

Quality assessment / Risk of bias analysis: The NOS items were used to assess the quality of the included studies. The risk of bias analysis were conducted by software Stata.12E.

Strategy of data synthesis: Qualitative analysis was performed by forest plot, while dose response was used for quantitative analysis.

Subgroup analysis: Subgroup analyses were performed according to the clinical characteristics of the study according to the size of the heterogeneity seen in the study.

Sensitivity analysis: Sensitivity analyses were performed by excluding each study individually and, if necessary, by trim-andfill method.

Language: English.

Country(ies) involved: China and Germany.

Keywords: HDL; Cholesterol Efflux Capacity; Cardiovascular disease; Metaanalysis.

Contributions of each author:

Author 1 - Wenke Cheng.

Email: cwk2517@163.com

Author 2 - Julia Boettner.

Author 3 - Tina Fischer-Schaepman.

Author 4 - Sarah Werner.

Author 5 - Angela Kricke.

Author 6 - Holger Thiele.

Author 7 - Petra Buettner.

Email: Petra.Buettner@medizin.uni-leipzig.de 